\title{
Paratiroidectomía transoral endoscópica por acceso vestibular oral. Cirugía de paratiroides sin cicatriz. Primer caso realizado en Sudamérica
}

\author{
Patricio Cabané T. ${ }^{1}$, Patricio Gac E. ${ }^{1}$, Daniel Rappoport W. ${ }^{1}$, \\ Francisco Rodríguez M. ${ }^{1}$ y Joaquín Ulloa S. ${ }^{1}$
}

\section{Transoral endoscopic parathyroidectomy vestibular approach (TOEPVA)}

Introduction: The approach of choice of the thyroid and parathyroid glands, since its description, has been the transverse cervicotomy. In hyperparathyroidism, with the optimization of the preoperative localization methods of the anomalous glands, minimally invasive open techniques have been created, with a better aesthetic result and a shorter recovery time. With the aim of eliminating external scars, a new surgical technique has been implemented that uses endoscopic approaches through natural orifices (NOTES). In recent years, a transoral endoscopic thyroidectomy and parathyroidectomy technique through incisions in the oral vestibule has been known. Clinical case: A 65-year-old patient with primary hyperparathyroidism with right upper hyperfunctioning nodule who underwent partial transoral endoscopic parathyroidectomy due to vestibular approach. Results: The procedure is performed without incidents with good postoperative evolution of the patient. Discussion: Transoral endoscopic parathyroidectomy by vestibular approach (TOEPVA) has proven to be a fairly safe technique, with results similar to open technique, but without leaving visible scars.

Key words: hyperparathyroidism; parathyroidectomy; minimally invasive surgical procedures.

\section{Resumen}

Introducción: El abordaje de elección de la glándula tiroides y paratiroides, desde su descripción, ha sido la cervicotomía transversal. En el hiperparatiroidismo, con la optimización de los métodos de localización preoperatoria de las glándulas anómalas, se han creado técnicas abiertas mínimamente invasivas, con un mejor resultado estético y un menor tiempo de recuperación. Con el objetivo de eliminar las cicatrices externas se ha implementado una nueva técnica quirúrgica que utiliza abordajes endoscópicos a través de orificios naturales (NOTES). En los últimos años se ha hecho conocida una técnica de tiroidectomía y paratiroidectomía transoral endoscópica a través de incisiones en el vestíbulo oral. Caso clínico: Paciente de 65 años con hiperparatiroidismo primario con nódulo hiperfuncionante superior derecho que se somete a una paratiroidectomía parcial transoral endoscópica por abordaje vestibular. Resultados: El procedimiento se realiza sin incidentes con buena evolución posoperatoria de la paciente. Discusión: La paratiroidectomía transoral endoscópica por abordaje vestibular (TOEPVA) ha demostrado ser una técnica bastante segura, con resultados similares a los de la técnica abierta, pero sin dejar cicatrices visibles.

Palabras clave: hiperparatiroidismo; paratiroidectomía; procedimientos quirúrgicos mínimamente invasivos.

\section{Introducción}

El abordaje de elección de la glándula tiroides y paratiroides, desde su descripción en 1880 por Theodor Kocher, ha sido la cervicotomía transversal anterior $^{1}$. Esta otorga una buena exposición, a través de un abordaje directo a la zona central del cuello. Lamentablemente, a pesar de un cierre meticuloso de la incisión, siempre quedará una cicatriz de proporciones variables en el cuello.

En Asia, una cicatriz cervical es considerada cosméticamente desfigurante, es por esto que en Tai-
Departamento de Cirugía Hospital Clínico Universidad de Chile.

Recibido el 12 de febrero y aceptado para publicación el 11 de junio de 2018.

Correspondencia a: Dr. Patricio Cabané T. pcabane@hcuch.cl 
landia, China, Corea y Japón se han intentado implementar nuevas técnicas; mínimamente invasivas y por accesos remotos, como el acceso transaxilar, el retroauricular o el transmamario. Cada una de estas técnicas han sido asociadas a morbilidades específicas que han impedido su adaptación global en el mundo ${ }^{2}$. En general, no se pueden realizar técnicas mínimamente invasivas en pacientes con cirugías previas, tumores de gran tamaño o sospecha de cáncer con diseminación regional. Otra consideración que hay que tener en cuenta, es que por lo general, las técnicas actuales por accesos remotos, permiten un acceso unilateral, necesitando dos abordajes (cicatrices) para realizar una cirugía que comprenda la zona cervical de forma bilateral.

Con el objetivo de eliminar completamente las cicatrices externas en el cuerpo se ha implementado una nueva técnica quirúrgica que utiliza abordajes endoscópicos a través de orificios naturales (NOTES). Esta técnica se ha adaptado para realizar abordajes cervicales en cirugía de tiroides y paratiroides ${ }^{3}$.

Basándose en los principios de las técnicas NOTES, apareció la cirugía endoscópica transoral de la glándula tiroides vía vestibular (TOETVA) y su modificación para la cirugía de paratiroides (TOEPVA). Se han publicado series de pacientes operados con esta última técnica con diagnóstico de hiperparatiroidismo con enfermedad uni o multiglandular ${ }^{4}$. Los resultados han sido alentadores, los

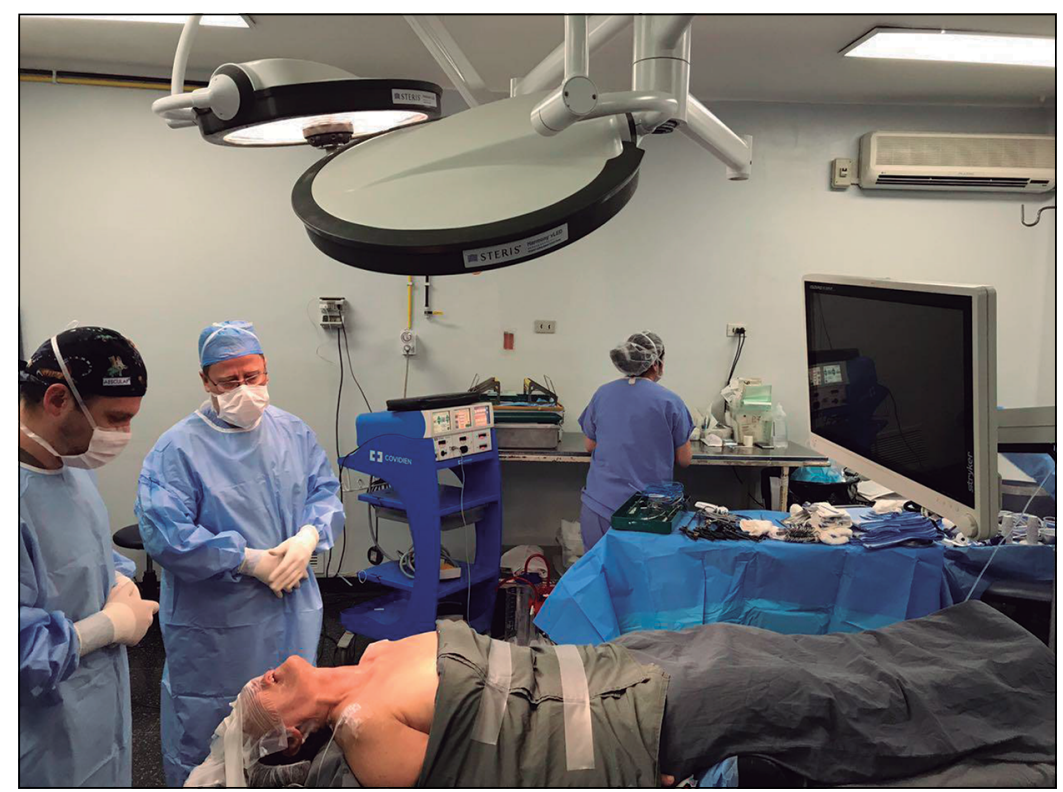

Figura 1. Paciente en decúbito supino con hiperextensión cervical y fijación craneal a la mesa operatoria. tiempos quirúrgicos pueden ser comparables con la técnica clásica abierta y la disección del tejido es mucho menor en comparación a las otras técnicas con abordajes remotos ${ }^{2}$.

René Gordillo de Ecuador publicó la primera tiroidectomía realizada de forma endoscópica vía vestíbulo oral en latinoamérica ${ }^{5}$.

A continuación presentamos el primer caso de un paciente con hiperparatiroidismo operado por un acceso transoral endoscópico por vestíbulo oral (TOEPVA) en Sudamérica.

\section{Caso clínico}

Paciente de 65 años de edad, de sexo femenino, con antecedentes de enfermedad de Basedow Graves tratada con radioyodo en su juventud e hipertensión arterial leve. Dentro de un estudio de rutina, presentó una hipercalcemia aislada que se estudió de forma más dirigida. Los exámenes que la paciente presentó fueron los siguientes: calcemia de hasta $10,8 \mathrm{mg} / \mathrm{dl}$, fosfemia $2,1 \mathrm{mg} / \mathrm{dl}$, PTH intacta $230 \mathrm{pg} / \mathrm{ml}$, Vit D normal y calciuria elevada. Frente a esto, se decidió complementar estudio con imágenes. Se realizó una ultrasonografía cervical que mostró un nódulo de 1,6 $\mathrm{cm}$ de diámetro mayor en relación a polo superior del lóbulo tiroideo derecho, sugerente de un adenoma de paratiroides superior derecha. También se realizó un cintigrama Sestamibi que confirmó una captación superior derecha.

Se analizó el caso en equipo y debido a que se trataba de una paciente de cuello corto, sin comorbilidades mayores, sin cirugías previas ni nódulos tiroideos asociados y con una lesión identificada claramente en los estudios preoperatorios; se decidió plantear una cirugía con técnica TOEPVA.

Se planificó la cirugía con el consentimiento informado de la paciente, considerando que se realizaría una técnica nueva, con la posibilidad de convertir a un acceso abierto clásico. La paciente fue sometida a una paratiroidectomía parcial endoscópica vía vestíbulo oral.

\section{Técnica quirúrgica}

En pabellón, bajo anestesia general, se posiciona a la paciente en decúbito supino con hiperextensión cervical. Se realiza intubación nasotraqueal y también fijación craneal a la mesa operatoria. Se completa la preparación con un aseo de cavidad oral con clorhexidina acuosa (Figura 1).

Se procede a realizar una incisión horizontal en el vestíbulo oral bajo el labio inferior de $11 \mathrm{~mm}$ aproximadamente. Se realiza una dilatación roma 


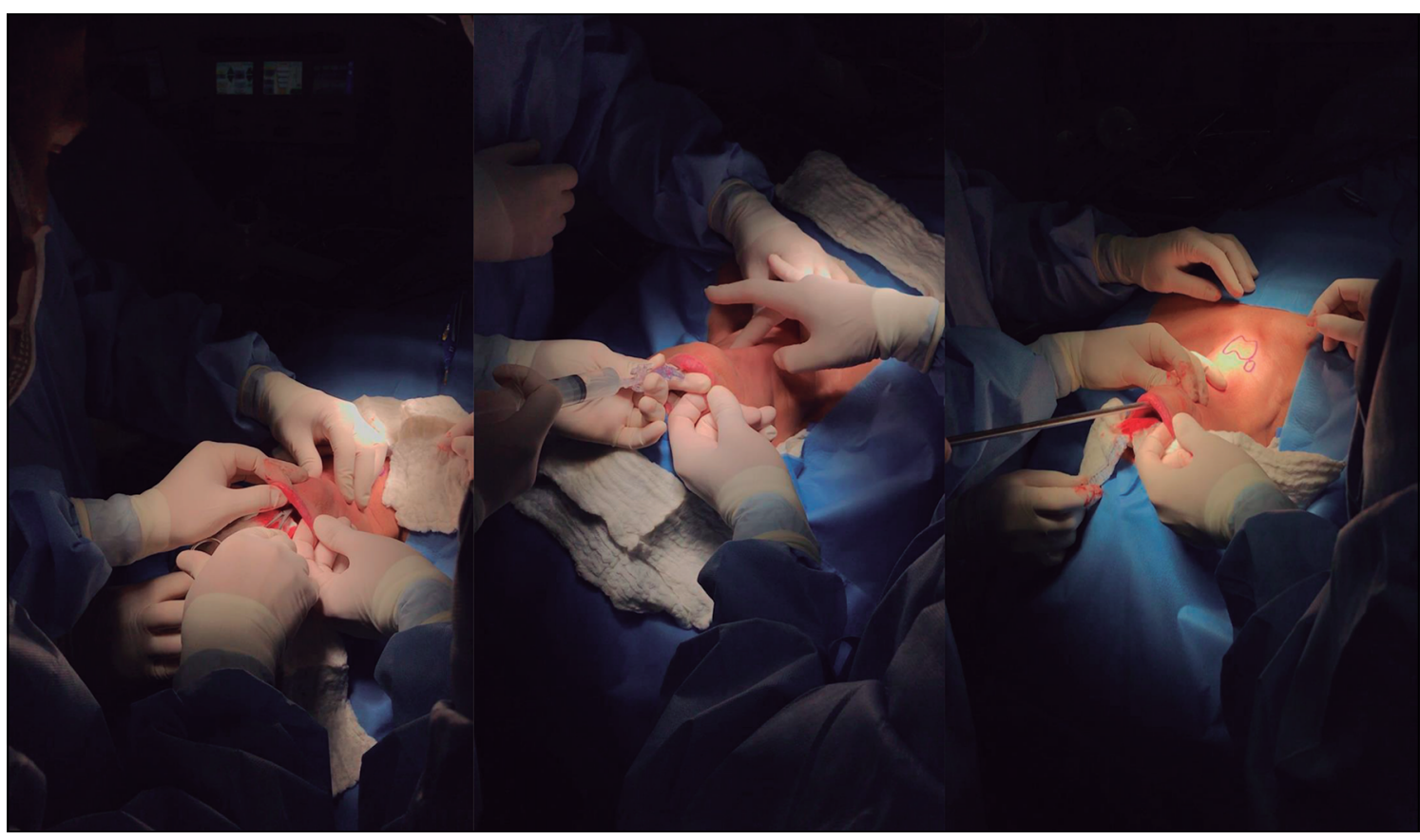

Figura 2. Dilatación roma con una pinza Kelly hasta espacio subperióstico premental (a). Hidrodisección con aguja de Veress hasta espacio supraesternal (b) Dilatación subcutánea con tunelizador romo (c). con una pinza kelly hasta el espacio subperióstico premental. Se continúa con una hidrodisección con aguja de Veress (30-40 $\mathrm{ml}$ de solución fisiológica con epinefrina), hasta espacio supraesternal, por línea media. Dilatación subcutánea con tunelizador romo (Figuras 2 a, b y c).

Inserción de trocar de $11 \mathrm{~mm}$ e insuflación con $\mathrm{CO}_{2}$ a $6 \mathrm{mmHg}$. Luego se realizan dos incisiones de $5 \mathrm{~mm}$ bajo borde de labio inferior, a la altura de los caninos, y se insertan trócares de $5 \mathrm{~mm}$ (Figura 3).

Con instrumental de laparoscopia tradicional se realiza disección del espacio subplatismal hacia caudal. Se identifican músculos pretiroideos y músculos esternocleidomastoideos como límites laterales. Se accede por rafe medio con hook y pinza armónica hasta identificar superficie tiroidea.

Retracción de músculos pretiroideos derechos con punto transcutáneo de seda 0 (Figura 4). Disección extracapsular de lóbulo tiroideo derecho. Liberación del lóbulo piramidal para mejorar movilidad del lóbulo derecho. Se identifica glándula paratiroides superior derecha aumentada de tamaño y consistencia en relación a arteria tiroidea inferior derecha (Figura 5). Disección extracapsular de paratiroides. Identificación y sección del pedículo con pinza armónica.

Extracción de paratiroides en bolsa (Figura 6). Se envía muestra a biopsia contemporánea certificando que corresponde a una glándula paratiroides altera-

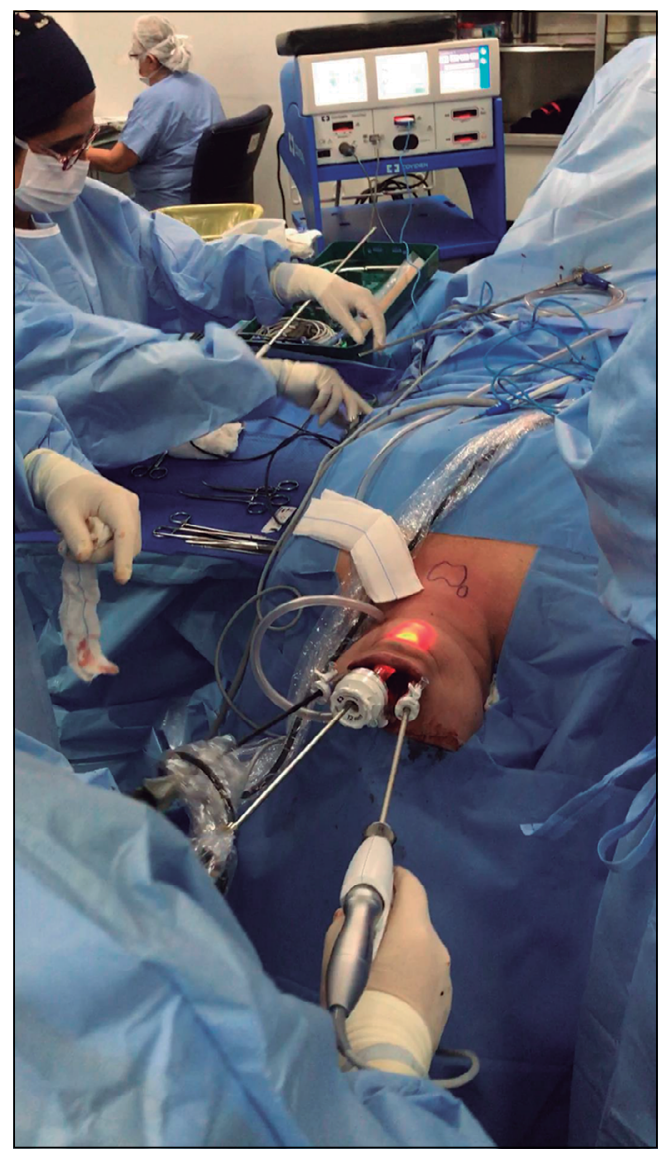

Figura 3. Inserción de trocar de $11 \mathrm{~mm}$ central y sobre caninos, dos trocares de $5 \mathrm{~mm}$. 


\section{CASOS CLÍNICOS}

Figura 4. Retracción de músculos pretiroideos derechos con punto transcutáneo de seda 0 .

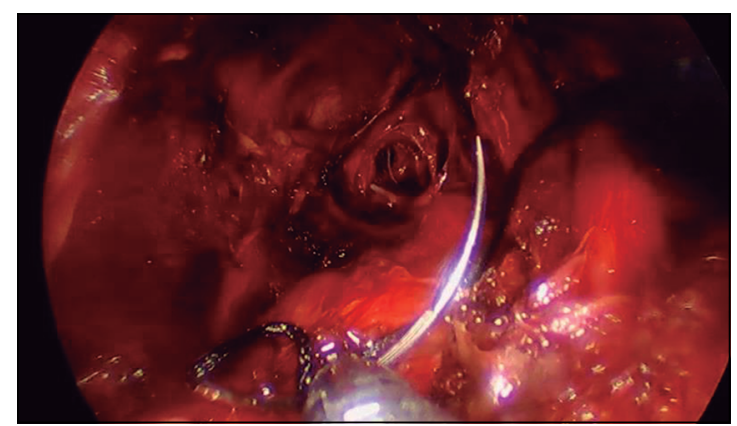

Figura 6. Extracción de paratiroides en bolsa.

Figura 7. Paciente a las 48 horas de la cirugía.

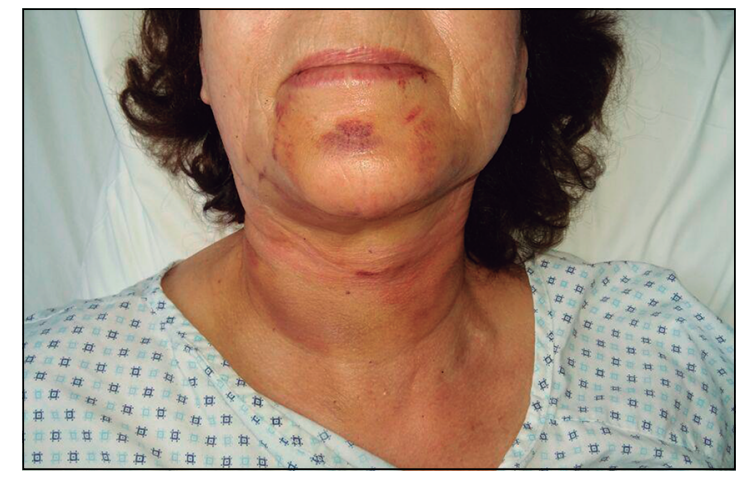

Figura 8. Paciente a los 30 días de la cirugía.

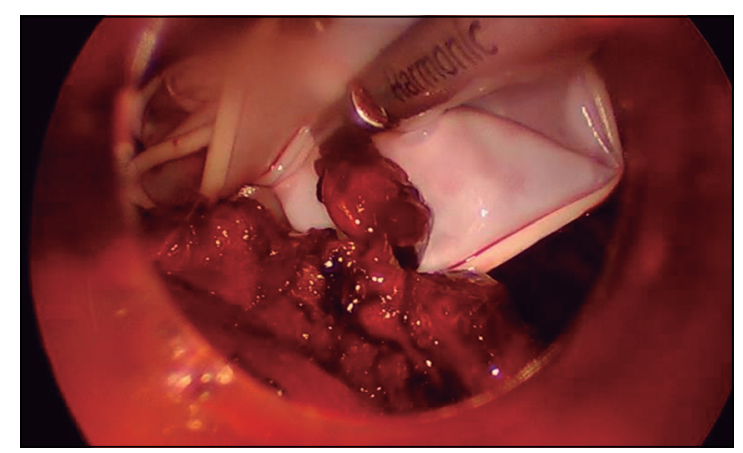

da. La medición de PTH intraoperatoria confirmó la resección del adenoma hiperfuncionante $(\mathrm{PTH}$ intraoperatoria Basal: $180 \mathrm{pg} / \mathrm{ml}$; PTH intraoperatoria $15 \mathrm{~min}: 42,6 \mathrm{pg} / \mathrm{ml}$; PTH intraoperatoria 30 $\min : 33,4 \mathrm{pg} / \mathrm{ml}$ ).

Se revisa la hemostasia de forma prolija. Se deja surgicel fibrilar ${ }^{\circledR}$ en el lecho operatorio. Cierre de músculos pretiroideos con puntos separados de vycril 3/0. Se retiran trócares bajo visión directa. Cierre de incisiones vestibulares en dos planos con puntos separados de vycril 3/0 y 4/0. Se realiza curación compresiva en mentón y región submentoniana.

\section{Resultados}

La cirugía se realizó sin incidentes. En el periodo posoperatorio, la paciente evolucionó con dolor leve en la zona de las incisiones y en el trayecto del abordaje. El dolor se manejó con antiinflamatorios no esteroidales con buena respuesta. Se identificó una normalizacion de la calcemia en exámenes sanguíneos posteriores a la cirugía. Se realizó el alta hospitalaria a las $48 \mathrm{~h}$ desde la cirugía. Al momento del alta la paciente solo presentaba equimosis leve en la región operatoria (Figura 7). La paciente nunca presentó disfonía, parestesia de territorio de nervio mental ni desviación de la comisura labial; tampoco presentó hematoma en la cavidad oral en relación a las incisiones ni signos de infección.

Dos meses después de la cirugía, la paciente se encuentra en buenas condiciones generales, sin complicaciones asociadas a la cirugía (Figura 8).

\section{Discusión}

Cuando hablamos de cirugía en el hiperparatiroidismo primario, históricamente la cirugía consistía en explorar de forma bilateral a través de una inci- 
sión cervical las cuatro glándulas paratiroides con un rango alto de curabilidad, de hasta un $97 \%{ }^{6}$.

Con la optimización de los métodos de localización preoperatoria de las glándulas anómalas, como la ultrasonografía de alta resolución o el cintigrama Sestamibi con Tecnecio 99m, se ha vuelto posible solo explorar y resecar la glándula afectada ${ }^{7}$. Monitorizar la paratohormona intraoperatoria ha dado pie para técnicas abiertas mínimamente invasivas (MIVAT), con un mejor resultado estético y un menor tiempo operatorio y de recuperación posoperatoria ${ }^{4,8}$. Realizar una incisión pequeña cervical anterior y de forma asistida por un endoscopio, identificar la glándula afectada y resecarla; ha sido en las últimas décadas el método más utilizado en Estados Unidos, siendo aplicable de forma ambulatoria en casos seleccionados ${ }^{9,10}$.

Gagner en 1996 fue el primer cirujano en realizar una cirugía cervical vía endoscópica de forma satisfactoria. La cirugía consistió en una paratiroidectomía parcial por un hiperparatiroidismo primario abordado por cuatros trócares de $5 \mathrm{~mm}$ instalados a $1 \mathrm{~cm}$ de la escotadura yugular y de la clavícula en la zona cervical anterior ${ }^{11}$.

No fue hasta el año 2009 que, Karakas et al, con el afán de eliminar las incisiones externas, reportó por primera vez una técnica NOTES en una cirugía de paratiroides. A esta técnica la llamó TOPP (Transoral partial parathyroidectomy), donde realizaba un abordaje cervical a través del piso de la boca por debajo de la lengua. Un par de años después publicó una serie de 5 casos de hiperparatiroidismo primario abordados con la misma técnica TOPP, y concluyó que no era una buena técnica por las complicaciones que presentaban los pacientes, como hematoma del piso de la boca, infección y posible lesión del nervio hipogloso ${ }^{7,4}$.

Anuwong et al, el año 2015 publicó, con buenos resultados, 60 casos de tiroidectomías vía transoral a través de 3 incisiones en el vestíbulo oral (TOETVA) ${ }^{12}$ y no a través del piso de la boca.
Desde entonces, se han publicado distintos trabajos mostrando los buenos resultados de este abordaje en cirugía de paratiroides ${ }^{2,4}$.

Como se pudo ver en nuestro caso, utilizando instrumental de laparoscopia convencional e insuflando con $\mathrm{CO}_{2}$ el espacio premandibular, se logra realizar un procedimiento bastante seguro y con poca destrucción del tejido a nivel del lecho tiroideo.

La técnica TOEPVA presenta resultados similares a los de la técnica abierta, pero a diferencia de esta última, no deja cicatriz cervical, presenta menos complicaciones en manos experimentadas y permite abordar el lecho de forma bilateral ${ }^{6,13}$. El tiempo quirúrgico sigue siendo mayor.

La técnica endoscópica por vestíbulo oral para paratiroidectomías ha tenido un rápido crecimiento en el último tiempo, siendo adoptada por grandes centros americanos. En algunas partes se ha intentado complementar la técnica TOEPVA con la utilización de la cirugía robótica mejorando, en parte, la disección debido a los movimientos articulares que el robot proporciona.

Si bien los resultados son alentadores, cabe destacar que se deben elegir con bastante mesura los pacientes candidatos para esta técnica y que también, es necesario un mayor número de casos para ofrecer cifras que tengan un valor estadístico.

\section{Responsabilidades éticas}

Protección de personas y animales. Los autores declaran que para esta investigación no se han realizado experimentos en seres humanos ni en animales.

Confidencialidad de los datos. Los autores declaran que en este artículo no aparecen datos de pacientes.

Derecho a la privacidad y consentimiento informado. Los autores declaran que en este artículo no aparecen datos de pacientes.

Conflictos de interés: No hay.

\section{Referencias}

1. Russell J, Clark J, Noureldine S, Anuwong A, Al Khadem MG, Kim HY, et al. Transoral thyroidectomy and parathyroidectomy-a North American series of robotic and endoscopic transoral approaches to the central neck. Oral Oncol. 2017;71:75-80.

2. Udelsman R, Anuwong A, Oprea AD, Rhodes A, Prasad M, Sansone M, et al.
Trans-oral vestibular endocrine surgery: a new technique in the United States. Ann Surg. 2016;264:13-6.

3. Dionigi G, Chai YJ, Tufano RP, Anuwong A, Kim YH. Transoral endoscopic thyroidectomy via a vestibular approach: why and how? Endocrine 2018;59:275-9.

4. Sasanakietkul T, Jitpratoom P, Anuwong A. Transoral endoscopic parathyroidectomy vestibular approach: a novel scarless parathyroidsurgery. Surg Endosc. 2017;31:3755-63.

5. René Gordillo V, Wilson Vásquez I, Amber Andrade C. Tiroidectomía transoral endoscópica por abordaje vestibular (TOETVA): reporte del primer caso en humanos realizado en Latinoamérica. Rev Chil Cir. 2017;69:604.

6. Anuwong A, Sasanakietkul T, Jitpratoom P, Ketwong K, Kim HY, Dionigi G, et al. 


\section{CASOS CLÍNICOS}

Transoral endoscopic thyroidectomy vestibular approach (TOETVA): indications, techniques and results. Surg Endosc. 2018;32:456-65

7. Karakas E, Steinfeldt T, Gockel A, Westermann R, Sommer F, Richard HR, et al. Transoral thyroid and parathyroid surgery. Surg Endosc. 2010;24:1261-7.

8. Miccoli P, Bendinelli C, Conte M, Pinchera A, Marcocci C. Endoscopic parathyroidectomy by a gasless approach. J Laparoendosc Adv Surg Tech A. 1998;8:189-94.
9. Carling T, Donovan P, Rinder C, Udelsman R. Minimally invasive parathyroidectomy using cervical block: reasons for conversion to general anesthesia. Arch Surg. 2006;141:401-4.

10. Udelsman R, Lin Z, Donovan P. The superiority of mini-mally invasive parathyroidectomy based on 1650 consecutive patients with primary hyperparathyroidism. Ann Surg. 2011;253:585-91.

11. Gagner M. Endoscopic subtotal parathyroidectomy in patients with primary hyperparathiroidism. Br J Surg. 1996;83:875.

12. Anuwong A. Transoral endoscopic thyroidectomy vestibular approach: a series of the first 60 human cases. World $\mathrm{J}$ Surg. 2016;40:491-7.

13. Jitpratoom P, Ketwong K, Sasanakietkul $\mathrm{T}$, Anuwong A. Transoral endoscopic thyroidectomy vestibular approach (TOETVA) for Graves' disease: a comparison of surgical results with open thyroidectomy. Gland Surg. 2016;5:54652. 